\title{
Slowdown of the Electronic Relaxation Close to the Mott Transition
}

\author{
Sharareh Sayyad and Martin Eckstein \\ Max Planck Institute for the Structure and Dynamics of Matter, 22761 Hamburg, Germany \\ and University of Hamburg-CFEL, 22761 Hamburg, Germany
}

(Received 13 January 2016; published 24 August 2016)

\begin{abstract}
We investigate the time-dependent reformation of the quasiparticle peak in a correlated metal near the Mott transition, after the system is quenched into a hot electron state and equilibrates with an environment which is colder than the Fermi-liquid crossover temperature. Close to the transition, we identify a purely electronic bottleneck time scale, which depends on the spectral weight around the Fermi energy in the bad metallic phase in a nonlinear way. This time scale can be orders of magnitude larger than the bare and renormalized electronic hopping time, so that a separation of electronic and lattice time scales may break down. The results are obtained using nonequilibrium dynamical mean-field theory and a slave-rotor representation of the Anderson impurity model.
\end{abstract}

DOI: 10.1103/PhysRevLett.117.096403

When the Mott metal-insulator transition [1] is approached from the metallic side, a narrow quasiparticle band emerges at the Fermi energy, and spectral weight is transferred into the Hubbard bands. This behavior, which is observed in a large class of materials, is a paradigm manifestation of many-body correlations, and its theoretical description has been a major success of dynamical meanfield theory (DMFT) [2,3]. By means of photoexcitation, metallic phases in Mott insulators can be induced on femtosecond time scales [4-6], which provides an intriguing example for ultrafast switching material properties. While it is well understood that an intense laser pulse can rapidly promote electrons to effective temperatures of several $1000 \mathrm{~K}$ and thus lead to a partial melting of the Mott gap [5], the equilibrium properties of such a hightemperature state would correspond to a bad metal rather than a Fermi liquid $[7,8]$. It thus remains a fundamental question, with immediate importance for understanding the transport properties of photoexcited metallic states, how fast coherent quasiparticles can be formed as the excitation energy is passed from the electrons to the lattice.

Naively one may expect that the electrons in a metal thermalize to a quasiequilibrium state almost instantly after the excitation, and quasiparticles are formed as soon as the effective temperature is low enough. The relevant time scale for this process would then be set by the electronlattice relaxation. In this work we show that a rapid thermalization can fail even in the metallic phase. While thermalization can be understood within a quasiparticle picture (from a kinetic equation), the latter provides no clue about the time scale for the evolution of the density of states itself, as long as quasiparticles are not yet well defined. Considerable progress in describing the dynamics of Mott insulators has been made using nonequilibrium DMFT [9], but a study of the correlated metal close to the Mott transition has remained elusive. Although the quasiparticle peak within DMFT corresponds to the Kondo resonance in an effective impurity model [2], its formation in time can be entirely different from the buildup of Kondo screening [10-15], because the spectral weight responsible for the Kondo screening is formed self-consistently in DMFT. Impurity solvers such as higher-order strong-coupling expansions [16], Monte Carlo techniques [17], or density-matrix renormalization group [18], have not yet reached sufficiently long times in this parameter regime.

In equilibrium, the slave-rotor approach developed by Florens and Georges $[19,20]$ provides an intuitive semianalytical understanding of the Mott transition, by representing electrons in terms of a quantum rotor (charge) and a spinful fermion. In this Letter we solve the coupled spinon and rotor equations out of equilibrium, and show that the two partial degrees of freedom become almost decoupled during the evolution. As a consequence, bad metallic behavior prevails in a photoexcited state over times which can be orders of magnitude longer than the electron hopping, and therefore even become comparable to the electron-phonon relaxation time.

Model.-We study the particle-hole symmetric Hubbard model

$$
\begin{aligned}
H= & -J(t) \sum_{\langle i j\rangle, \sigma}\left(c_{i \sigma}^{\dagger} c_{j \sigma}+\text { H.c. }\right) \\
& +U \sum_{i}\left(n_{i \uparrow}-\frac{1}{2}\right)\left(n_{i \downarrow}-\frac{1}{2}\right),
\end{aligned}
$$

where $J(t)$ is the time-dependent hopping amplitude, $U$ is the on-site Coulomb repulsion, $c_{i \sigma}$ and $c_{i \sigma}^{\dagger}$ are electron annihilation and creation operators for spin $\sigma \in\{\uparrow, \downarrow\}$ on site $i$, and $n_{i \sigma}=c_{i \sigma}^{\dagger} c_{i \sigma}$. To study the time-dependent formation of quasiparticles, we initially prepare the system in the atomic limit $(J=0)$, and rapidly turn on the hopping 
to a value $J_{0}>0$. (In the following, $J_{0}$ and $\hbar / J_{0}$ set the energy and time unit, respectively, and the ramp-on profile is given by $J(t)=J_{0}\left[1-\cos \left(\pi t / t_{c}\right)\right] / 2$ for $0 \leq t \leq t_{c}=$ 2.5.) The model is solved within nonequilibrium DMFT [9] on a Bethe lattice with half-bandwidth $D=2 J_{0}$; i.e., it is mapped onto an Anderson impurity problem with selfconsistently determined hybridization function $\Delta\left(t, t^{\prime}\right)=$ $J(t) G_{\mathrm{loc}}\left(t, t^{\prime}\right) J\left(t^{\prime}\right)$ [2], where $G_{\mathrm{loc}}\left(t, t^{\prime}\right)=-i\left\langle T_{\mathcal{C}} c(t) c^{\dagger}\left(t^{\prime}\right)\right\rangle$ is the local contour-ordered Green's function [21].

To solve the dynamics of this Anderson model, we employ the $\mathrm{U}(1)$ slave-rotor representation [20]. The impurity operators $\left(c_{\sigma}, c_{\sigma}^{\dagger}\right)$ are substituted by $c_{\sigma}^{\dagger}=f_{\sigma}^{\dagger} e^{i \theta}$, where $f_{\sigma}^{\dagger}$ is a fermion and $\theta \in[0,2 \pi)$ is a quantum rotor variable. A constraint $L=\sum_{\sigma} f_{\sigma}^{\dagger} f_{\sigma}-1$ on the angular momentum $L=i \partial_{\theta}$ of the rotor removes unphysical states from the Hilbert space. With this, the interaction Hamiltonian is determined only by the rotor, $H_{U}=$ $U L^{2}$, while $f_{\sigma}$ represents a chargeless fermion (spinon). Furthermore, the rotor is replaced by a bosonic field $X=e^{i \theta}$ with the constraint $|X(t)|^{2}=1$. The dynamics of the impurity model is then analyzed in terms of contourordered rotor and spinon Green's functions

$$
\begin{aligned}
& G_{X}\left(t, t^{\prime}\right)=-i\left\langle T_{\mathcal{C}} X(t) X^{*}\left(t^{\prime}\right)\right\rangle, \\
& G_{f}\left(t, t^{\prime}\right)=-i\left\langle T_{\mathcal{C}} f_{\sigma}(t) f_{\sigma}^{*}\left(t^{\prime}\right)\right\rangle,
\end{aligned}
$$

where $G_{X}$ has a direct relation to the local charge susceptibility [20], and subsequently the electron's Green's function is obtained by $G_{\mathrm{loc}}\left(t, t^{\prime}\right)=i G_{f}\left(t, t^{\prime}\right) G_{X}\left(t, t^{\prime}\right)$. The model can be solved exactly when the spin-degeneracy $N$ and the number of rotor flavors $M$ is increased from $N=2$ and $M=1$ to infinity, keeping the ratio $\mathcal{N}=N / M$ fixed [20], and this limit provides a qualitatively correct description of the metal-insulator transition. The resulting integral equations correspond to a reformulation of the Ref. [20] within the Keldysh framework and are given in the Supplemental Material [22].

Results. - In Fig. 1(a), we plot the electronic density of states for three temperatures at $U / J_{0}=4$ in equilibrium $\left(J(t)=J_{0}\right)$. The metal insulator transition endpoint is at $U_{c} \approx 4.69$. Below a temperature $T^{*} \approx 0.2$, a quasiparticle peak emerges at the Fermi energy, while for $T>T^{*}$ the system is in a bad metallic state with a pseudogap at the Fermi energy. At intermediate values of $U$ and in an isolated system, the quench would lead to a highly excited electronic state which thermalizes within a few $1 / J_{0}$ to an effective temperature above the Fermi-liquid crossover $T^{*}$, which is also confirmed by the slave-rotor calculations. A similar fast thermalization at very high electronic temperatures was obtained in the Hubbard model after excitation with an electric field pulse [23], so that the quenched state is a good representation of a hot-electron state reached after strong photoexcitation [22].
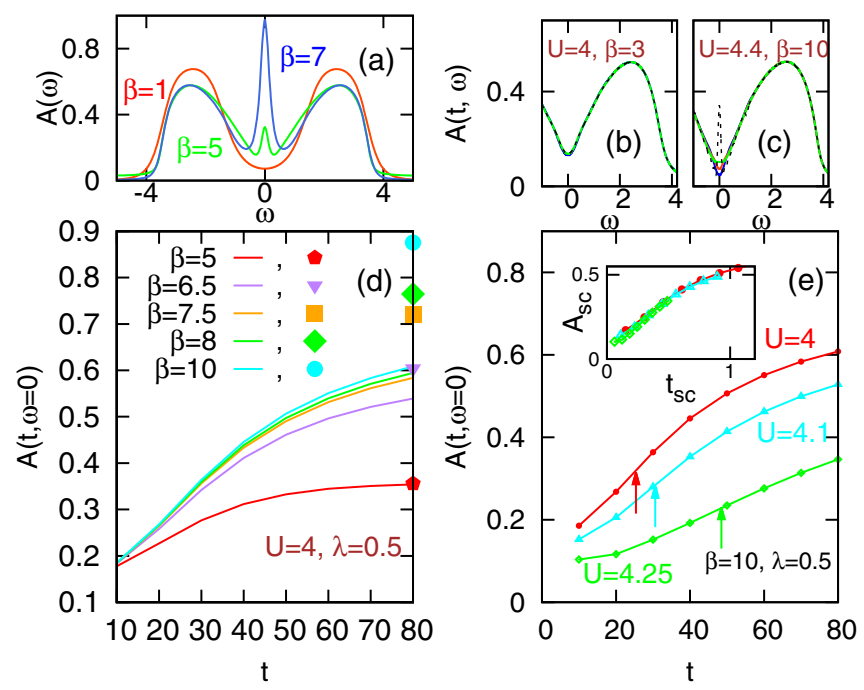

FIG. 1. (a) Equilibrium density of state $A(\omega)$ for three different temperatures throughout the metal-insulator crossover at $U=4$. (b),(c) $A(\omega)$ in equilibrium (dashed line) compared to the timedependent spectral function $A(t, \omega)$ for $\lambda=0.5$ at times $t=20$ (blue), $t=50$ (red), and $t=80$ (green). (d) The height of the quasiparticle peak $A(t, \omega=0)$ as a function of time for $U=4$, bath temperatures $\beta=5,6.5,7.5,8,10$ (from bottom to top) and $\lambda=0.5$. Symbols on the right vertical axis correspond to the equilibrium value $A(\omega=0)$ at the same temperatures. (e) The height of the quasiparticle peak $A(t, \omega=0)$ as a function of time for $U=4,4.1,4.25$, bath temperature $\beta=10$, and $\lambda=0.5$. Arrows indicate the time $t_{\max }$ [Fig. 3(c)]. The inset of (e) plots the time-dependent spectral function $A_{\mathrm{sc}}\left(t_{\mathrm{sc}}\right)=A(t, \omega=0)$ as a function of rescaled time $t_{\mathrm{sc}}=t / \tau^{*}$ with arbitrary rescaling of the vertical axis (see main text).

In addition, the system is weakly coupled to a bosonic heat bath at low temperature $T=1 / \beta$, to cool down the electrons and form the Fermi liquid when $U / J_{0}$ is in the metallic phase. We treat this dissipative bath by an additional electron self-energy $\Sigma_{\text {bath }}\left(t, t^{\prime}\right)=\lambda D\left(t, t^{\prime}\right) G\left(t, t^{\prime}\right)$, where $D$ is the noninteracting bosonic Green's function with frequency $\omega_{0}=1$, and $\lambda$ is the coupling constant [24]. The coupling is small enough so that the effect of the bath on the electronic density of states is weak, and the bath provides only energy relaxation.

To track the time evolution of the system, we compute the time-dependent spectral function $A(t, \omega)=$ $-(1 / \pi) \operatorname{Im} \int_{0}^{t} d s G^{\mathrm{ret}}(t, t-s) e^{i \omega s}$. For bath temperatures $T>T^{*}, A(t, \omega)$ is almost indistinguishable from the equilibrium spectrum $A(\omega)$ at temperature $T$ already at early times $t=20$ [Fig. 1(b)]. For lower temperature, however, only the Hubbard bands are rapidly retrieved, while the formation of the quasiparticle peak remains incomplete even for times larger than the inverse width of the peak [Fig. 1(c)]. The slow dynamics is also clear from the time evolution of the spectral weight $A(t, \omega=0)$ [Fig. 1(d)]: For $T<T^{*}$, the equilibrium value $A(0)$ strongly increases with decreasing $T$, while 
the time-dependent value $A(t, 0)$ becomes almost independent of the bath temperature, indicating that the dynamics is governed by a bottleneck of electronic nature. The closer $U$ is to the critical value $U_{c}$, the less metallic is the transient state [Fig. 1(e)]. (The presence of such a bottleneck makes it impossible to extrapolate the data $A(t, \omega=0)$ to the final equilibrium value from the early times around the bottleneck.) We note that qualitatively the same behavior is found using the noncrossing impurity solver [16], although the latter is not quantitatively accurate around the metalinsulator transition, and $U_{c}$ is reduced [22]. In the following, we will use the slave-rotor language to identify a purely electronic crossover time scale [c.f. Eq. (4) below], which captures the slow-down of the relaxation.

Despite the well-known equilibrium physics of the Hubbard model, the slave-rotor language exhibits a nontrivial spinon response in the crossover regime. Figures 2(a)-2(c) show the spectral functions $A_{X, f}(\omega)=$ $-(1 / \pi) \operatorname{Im} \int d s G_{X, f}^{\mathrm{ret}}(s) e^{i \omega s}$ in equilibrium. At high temperature, the rotor has spectral weight around the Hubbard bands, and the spinon peak is broadened due to the interaction with the charge fluctuations [Fig. 2(a)]. Below the crossover [Fig. 2(c)], the rotor develops lowenergy spectral weight, which implies the formation of the quasiparticle peak [20]. In the intermediate temperature regime, however, the spinon and rotor become energetically weakly coupled, and $A_{f}(\omega)$ develops into a narrow Lorentzian peak [Fig. 2(b)]. The width $\Gamma$ of the Lorentzian defines a time scale $\tau_{\mathrm{eq}}(T)=1 / \Gamma$, which has a clear maximum $\tau_{*}$ as a function of temperature in the metal-insulator crossover [Fig. 2(d)] [25]. To characterize
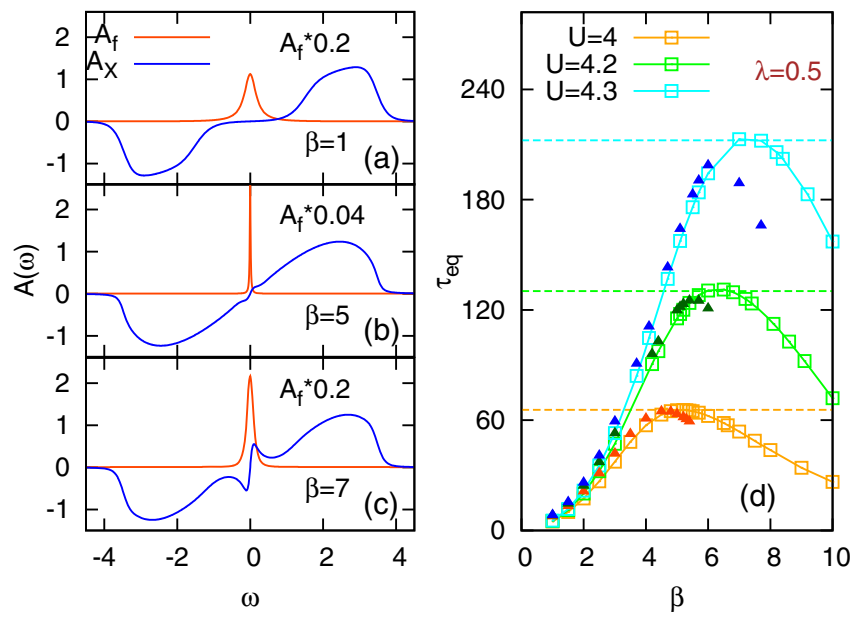

FIG. 2. (a)-(c) Spinon $(f)$ and rotor $(X)$ spectral functions in equilibrium for $U=4$ and temperatures in the bad metal regime $[\beta=1$, (a) $]$, the crossover $[\beta=5$, (b)] and the metallic phase ( $[\beta=7$, (c)]. (d) The spinon lifetime (inverse width of the peak) as a function of $\beta$ for different values of $U$. Triangular points are calculated using the approximate expression Eq. (4). Dashed lines indicate the maximum spinon lifetime $\tau_{\max }$ during the relaxation process (see main text and Fig. 3). the time evolution, we plot $G_{f}^{\text {ret }}(t, t-s)$ as a function of time difference $s$ for various $t$ (Fig. 3). A narrow peak in $A_{f}(\omega)$ corresponds to a slow decay of $G_{f}$ as a function of $s$, so that we can define a nonequilibrium spinon lifetime by

$$
\tau_{\mathrm{ne}}^{-1}(t)=-\partial_{s} G_{f}^{\mathrm{ret}}(t, t-s) /\left.G_{f}^{\mathrm{ret}}(t, t-s)\right|_{s=s_{0}}
$$

for some fixed time $s_{0}$. (For a Lorentzian peak, $\tau$ is the inverse width). The time $\tau_{\text {ne }}$ first increases with $t$ and then decreases, tracking the evolution of $\tau_{\text {eq }}(T)$ a function of temperature [Fig. 3(b)]. We then find that, for a given coupling to the bath, the maximum of $\tau_{\mathrm{ne}}(t)$ as a function of time $\left(\tau_{\max }\right)$ coincides with the crossover scale $\tau_{*}$ [dashed lines in Fig. 2(d)], and moreover, this value is reached at a time $t_{\max }$ proportional to $\tau_{*}$ [Fig. 3(c)]. The electronic spectral function at $t=t_{\max }$ is at the onset of quasiparticle formation [arrows in Fig. 1(e)]. This demonstrates that the long lifetime of the spinon provides a bottleneck time for the relaxation in the crossover regime.

In Fig. 1(e) (and the inset) we show that $A(t, \omega=0)$ for various values of $U$ in the crossover regime can be roughly collapsed on each other when the time axis is rescaled by this crossover scale $\tau_{*}$; i.e., to this extent $\tau_{*}$ determines also the slowdown of the electronic relaxation. Furthermore, although properties of $A_{f}(\omega)$ are not simply reflected in the equilibrium single-particle properties, one can approximately express the timescale $\tau_{*}$ in terms of the electronic degrees of freedom. The width of a sharp resonance in $A_{f}(\omega)$ is given by the imaginary part of the self-energy $\Sigma_{f}$,

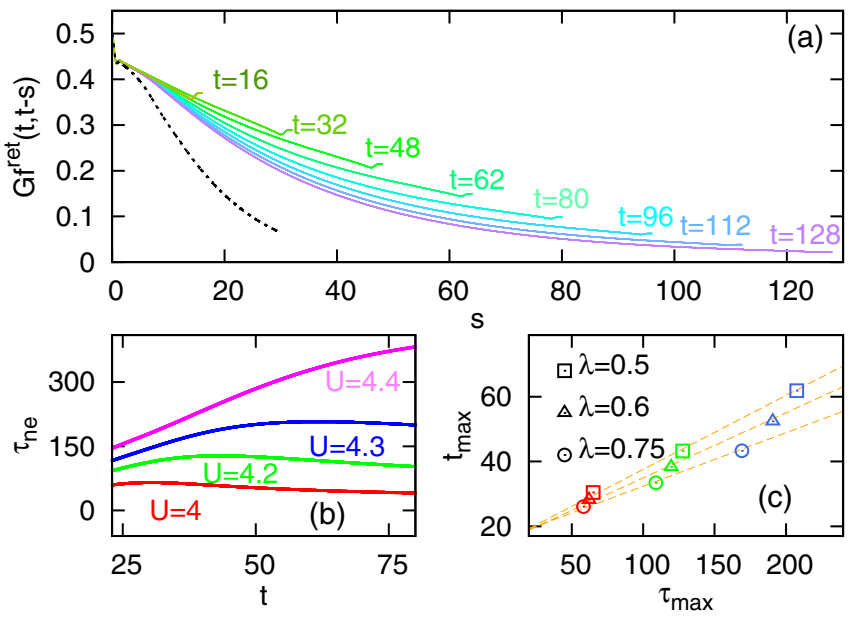

FIG. 3. (a) Retarded spinon Green's function $G_{f}^{\text {ret }}(t, t-s)$ as a function of relative time $s$ for various different times $t(U=4$, $\beta=10, \lambda=0.5$ ), and in equilibrium (dashed line). The slope decreases for $t \lesssim 32$ and increases for $t \gtrsim 32$. (b) Inverse of the slope [Eq. (3)] as a function of $t$ for $\lambda=0.5, \beta=10, s_{0}=16$ and various values $U$ below the metal-insulator transition; $s_{0}=16$ is chosen large enough so that $G_{f}\left(t, t-s_{0}\right)$ reflects the low-energy part of the Green's function (the Lorentzian peak). (c) Crossover time $t_{\max }$ plotted against $\tau_{\max }$, where $\left(t_{\max }, \tau_{\max }\right)$ corresponds to the maximum of the curves $\tau_{\text {ne }}(t)$ in panel (b). 
which depends on $G_{X}$ and $\Delta$. In the crossover regime, we can, to a first approximation, relate the rotor $G_{X}$ to the electronic Green's function by $A(\omega)=\frac{1}{2} A_{X}^{\prime \prime}(\omega) \operatorname{coth}(\beta \omega / 2)$, by setting $A_{f}(\omega)=\delta(\omega)$ in the convolution $G_{\mathrm{loc}}\left(t, t^{\prime}\right)=$ $i G_{f}\left(t, t^{\prime}\right) G_{X}\left(t, t^{\prime}\right)$ [26]. Analytic continuation of $\Sigma_{f}\left(t, t^{\prime}\right)=$ $i \Delta\left(t, t^{\prime}\right) G_{X}\left(t^{\prime}, t\right)$ then gives

$$
\tau_{\mathrm{eq}}^{-1}(T)=-\Sigma_{f}^{\prime \prime}(\omega=0) \approx-\int d \omega \frac{\Delta^{\prime \prime}(\omega) A(\omega)}{\cosh (\omega / 2 T)^{2}},
$$

and $\tau_{*}=\tau_{\text {eq }}\left(T^{*}\right)$, which agrees well with the numerical result [Fig. 2(d)]. For the Bethe lattice $\Delta=J^{2} G_{\text {loc }}$, so that $\Delta^{\prime \prime}(\omega)=-\pi J^{2} A(\omega)$. Equation (4) implies a rather nontrivial relation between the nonequilibrium relaxation and the electronic properties. At $T^{*}$, the hyperbolic cosine function restricts the integral to values close to the pseudogap, where $A(\omega)$ is small. Since the endpoint of the metal-insulator transition temperature in the Hubbard model is remarkably small compared to the bare energy scales, $\tau_{*}$ becomes much longer than the bare hopping close to the transition $U=U_{c}$.

To further analyze the relaxation, one can check whether the rotor reaches a quasiequilibrium state while the spinon is slowly evolving, by testing whether the fluctuation-dissipation $G_{X}^{>}(\omega, t) / G_{X}^{<}(\omega, t)=e^{\beta_{\text {eff }} \omega}$ is satisfied. The latter would imply that an effective temperature $T_{\text {eff }}=1 / \beta_{\text {eff }}$ can be assigned to charge fluctuations [using the time-dependent Fourier transforms $G^{>,<}(\omega, t)=$ $\left.\int d s G^{>,<}(t, t-s) e^{i \omega s}\right]$. Figure 4(a), however, shows that a single charge temperature cannot be defined on the time scale of the simulation. While the occupation of highenergy fluctuations (the Hubbard bands) is small, the low energy part remains at an apparent higher temperature (the slope of lines for $\omega \gtrsim-0.8$ is slightly smaller than for $\omega \lesssim-0.8$ ); i.e., high-energy and low-energy charge fluctuations are not thermalized with each other. The lower
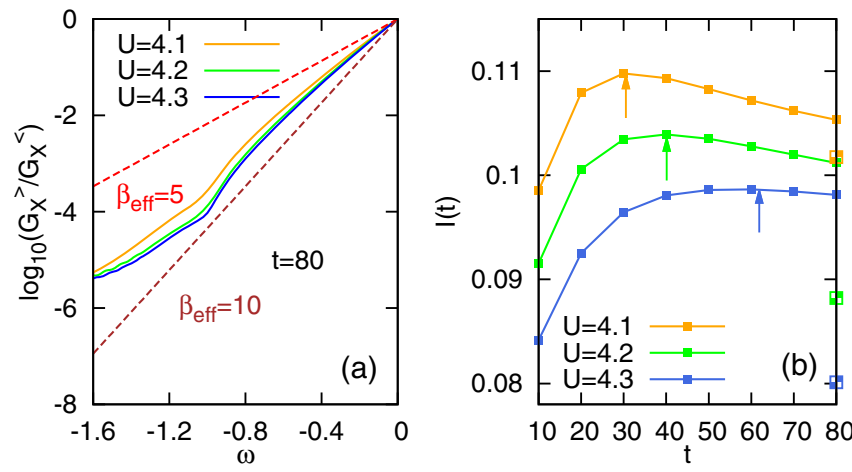

FIG. 4. (a) Test of the quasiequilibrium relation $G_{X}^{>}(\omega, t) / G_{X}^{<}(\omega, t)=e^{\beta_{\text {eff }} \omega}$ at $t=80$ for $U=4,4.2,4.3$ $(\lambda=0.75, \beta=10)$. (b) Integrated spectral weight $I(t)=$ $\int_{0}^{0.5} d \omega A_{X}(t, \omega)$ of the rotor at low energy. Square half-filled points at $t=80$ correspond to the equilibrium values. Arrows indicate the $t_{\max }$ [Fig. 3(c)]. effective temperature for larger $U$ may be related to the lowering of the crossover temperature with increasing $U$. Because of the coupling between the spinon and the rotor, the low-energy spectral weight of the rotor also reflects the nonmonotonic evolution of the spinon [Fig. 4(b)]: The increase of the spinon bandwidth for $t>t_{\max }$ leads to the transfer of the rotor spectral weight to higher energies, so that the integrated spectral weight $I(t)$ of the rotor in the low energy region $0<\omega<0.5$ has a maximum around $t=t_{\max }$.

Conclusion and discussion.-In conclusion, we have investigated how the electronic state close to the Mott transition in the Hubbard model relaxes from an excited hot-electron state towards the Fermi liquid. We found a bottleneck time of purely electronic nature, before which charge and electronic degrees of freedom remain in a nonthermal state and cannot be characterized by an effective temperature, and the formation of the quasiparticle band is incomplete. The electronic relaxation is related to the spinon lifetime $\tau_{*}$, and a simple estimate [Eq. (4)] in terms of the density of states around $\omega=0$ at the crossover temperature $T^{*}$ (the onset of quasiparticle formation) shows that this time can be much longer than the femtosecond hopping time, and thus violates the paradigm of rapid thermalization in a metal. The absence of quasiparticles implies long-lived bad metallic behavior, and should thus be observable also in optical experiments on materials like $\mathrm{LiV}_{2} \mathrm{O}_{4}$, which are metals close to the paramagnetic Mott transition [27,28]. We note that slow (or absent) formation of a quasiparticle band was also observed in simulations of a photodoped Mott insulator [24], but in this case the origin of the behavior is less clear because the final low temperature state is insulating.

It is important to note that $\tau_{*}$ characterizes the slow dynamics of the system around the crossover regime, but not necessarily the subsequent reshaping of the quasiparticle peak. Times larger than $t_{\max }$ cannot be studied systematically due to the increase of the numerical cost. The final formation of the quasiparticle peak might bring in another slow time scale related to the buildup of low energy spectral weight of the rotor. Furthermore, after quasiparticles are formed, slow dynamics can arise also from an ineffective coupling of heavy electrons to phonons [29]. The observed dynamical behavior arises from the DMFT self-consistency and is thus a lattice effect. In contrast, the buildup of the Kondo peak after a quench in the Anderson model is limited only by energy-time uncertainty [15] (i.e., the formation of the peak is complete after the inverse of its width), and this behavior is also reproduced by the slave-rotor method [22]. A natural question for future studies is thus whether a similar electronic bottleneck time may appear in multiband Hubbard models or the Kondo lattice model for heavy fermions, where localized $f$ or $d$ orbitals interacting with delocalized electrons give rise to the emergence of massive quasiparticles. In this context it is also interesting whether 
one can identify the small energy scale related to the spinon in numerically exact equilibrium calculations.

The authors would like to acknowledge fruitful discussions with A. Rosch, D. Golez, and Ph. Werner. The support by the Deutsche forschungsgemeinschaft within the sonderforschungsbereich 925 (project B4) is greatly acknowledged.

[1] M. Imada, A. Fujimori, and Y. Tokura, Rev. Mod. Phys. 70, 1039 (1998).

[2] A. Georges, G. Kotliar, W. Krauth, and M. J. Rozenberg, Rev. Mod. Phys. 68, 13 (1996).

[3] G. Kotliar, S. Y. Savrasov, K. Haule, V. S. Oudovenko, O. Parcollet, and C. A. Marianetti, Rev. Mod. Phys. 78, 865 (2006).

[4] S. Iwai, M. Ono, A. Maeda, H. Matsuzaki, H. Kishida, H. Okamoto, and Y. Tokura, Phys. Rev. Lett. 91, 057401 (2003).

[5] L. Perfetti, P. A. Loukakos, M. Lisowski, U. Bovensiepen, H. Berger, S. Biermann, P. S. Cornaglia, A. Georges, and M. Wolf, Phys. Rev. Lett. 97, 067402 (2006).

[6] D. Wegkamp, M. Herzog, L. Xian, M. Gatti, P. Cudazzo, C. L. McGahan, R. E. Marvel, R. F. Haglund, A. Rubio, M. Wolf, and J. Stähler, Phys. Rev. Lett. 113, 216401 (2014).

[7] J. Merino and R. H. McKenzie, Phys. Rev. B 61, 7996 (2000).

[8] X. Deng, J. Mravlje, R. Žitko, M. Ferrero, G. Kotliar, and A. Georges, Phys. Rev. Lett. 110, 086401 (2013).

[9] H. Aoki, N. Tsuji, M. Eckstein, M. Kollar, T. Oka, and Ph. Werner, Rev. Mod. Phys. 86, 779 (2014).

[10] F. B. Anders and A. Schiller, Phys. Rev. Lett. 95, 196801 (2005).

[11] D. Roosen, M. R. Wegewijs, and W. Hofstetter, Phys. Rev. Lett. 100, 087201 (2008).

[12] G. Cohen, E. Gull, D. R. Reichman, A. J. Millis, and E. Rabani, Phys. Rev. B 87, 195108 (2013).
[13] G. Cohen, E. Gull, D. R. Reichman, and A. J. Millis, Phys. Rev. Lett. 112, 146802 (2014).

[14] A. E. Antipov, Q. Dong, and E. Gull, Phys. Rev. Lett. 116, 036801 (2016).

[15] P. Nordlander, M. Pustilnik, Y. Meir, N. S. Wingreen, and D. C. Langreth, Phys. Rev. Lett. 83, 808 (1999).

[16] M. Eckstein and Ph. Werner, Phys. Rev. B 82, 115115 (2010).

[17] Ph. Werner, T. Oka, and A. J. Millis, Phys. Rev. B 79, 035320 (2009).

[18] F. A. Wolf, I. P. McCulloch, and U. Schollwöck, Phys. Rev. B 90, 235131 (2014).

[19] S. Florens and A. Georges, Phys. Rev. B 70, 035114 (2004).

[20] S. Florens and A. Georges, Phys. Rev. B 66, 165111 (2002).

[21] For an introduction and notation concerning nonequilibrium Green's functions, see Ref. [9].

[22] See Supplemental Material at http://link.aps.org/ supplemental/10.1103/PhysRevLett.117.096403 for a summary of the slave-rotor integral equations, and a comparision to results obtained from the non-crossing approximation, which includes Refs. [9,15,16,20,23].

[23] M. Eckstein and Ph. Werner, Phys. Rev. B 84, 035122 (2011).

[24] M. Eckstein and Ph. Werner, Phys. Rev. Lett. 110, 126401 (2013).

[25] Note that this development of the narrow spectral feature in the crossover regime due to the nonmonotonous coupling of the spinon and charge fluctuations is different from the zerotemperature singularities of the spinon and rotor Green's functions discussed in Ref. [20].

[26] For the analytic continuation of the slave-rotor equations to the real-frequency axis, see Ref. [20].

[27] J Matsuno, K Kobayashi, A Fujimori, L.F Mattheiss, and Y Ueda, Physica B (Amsterdam) 281-282B, 28 (2000).

[28] R. Arita, K. Held, A. V. Lukoyanov, and V. I. Anisimov, Phys. Rev. Lett. 98, 166402 (2007).

[29] J. Demsar, R. D. Averitt, K. H. Ahn, M. J. Graf, S. A. Trugman, V. V. Kabanov, J. L. Sarrao, and A. J. Taylor, Phys. Rev. Lett. 91, 027401 (2003). 